\title{
Characterization of Defects in Titanium Created by Hydrogen Charging
}

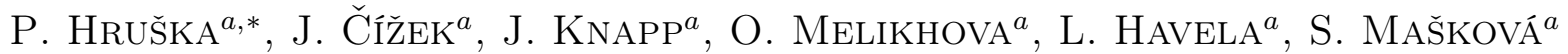 \\ AND F. LUKÁC̆ $\check{C}^{a, b}$ \\ ${ }^{a}$ Faculty of Mathematics and Physics, Charles University, V Holešovičkách 2, 18000 Prague, Czech Republic \\ ${ }^{b}$ Institute of Plasma Physics, Czech Academy of Sciences, Za Slovankou 3, 18200 Prague, Czech Republic
}

\begin{abstract}
Hydrogen interaction with vacancies in $\alpha$-Ti was investigated employing positron lifetime spectroscopy combined with $a b$ initio theoretical modeling of vacancy-hydrogen complexes. Ab initio modeling revealed that multiple hydrogen atoms up to 7 can be trapped at vacancies in the $\alpha$-Ti lattice. Trapped $\mathrm{H}$ atoms are located close to the nearest neighbor tetrahedral sites around the centre of vacancy. Lifetimes of positrons trapped at vacancies associated with various numbers of hydrogen atoms were calculated. Positron lifetime measurement of H-loaded $\alpha$-Ti samples revealed that phase transition into the hydride phase introduced dislocations. Vacancies were created by $\mathrm{H}$ loading as well and agglomerated into small vacancy clusters.
\end{abstract}

DOI: 10.12693/APhysPolA.132.1606

PACS/topics: 78.70.Bj, 61.72.-y

\section{Introduction}

Ti and Ti-based alloys exhibit high strength-to-weight ratio and good corrosion resistance. This makes them attractive for a wide range of applications including automotive and aerospace industries, power generation, medicine etc. [1]. Ti is an exothermic absorber of hydrogen [2]. $\mathrm{H}$ absorption in Ti leads to a degradation of its mechanical properties [3]. H-induced embrittlement of Ti is extensively studied since $\mathrm{Ti}$ and $\mathrm{Ti}$ alloys are exposed to hydrogen containing environment in many practical applications. There is an attractive interaction between absorbed $\mathrm{H}$ and open volume defects (vacancies, vacancy clusters, dislocations etc.) in many metals [4]. Hydrogen segregating at surfaces of open volume defects lowers their formation energy due to binding energy released when hydrogen is bound to the defect. Since this effect can be considered as analogy to surfactants lowering interfacial tension between two liquids or between a liquid and solid [5] a new term defactans $=$ DEFect ACTing AgeNTS was introduced for solutes segregating at open volume defects [6]. Because of its small size, high mobility and attractive interaction with open volume defects hydrogen seems to be a typical example of a defactant. Segregated hydrogen stabilizes open volume defects and increases their concentration [7-9]. For example the equilibrium concentration of vacancies becomes enhanced by many orders of magnitude in metals containing absorbed hydrogen [10, 11].

Employing the density functional theory (DFT) [12] the geometry of vacancy-hydrogen complexes and the number of $\mathrm{H}$ atoms which can be trapped in vacancy can be determined by ab initio calculations. Theoret-

*corresponding author; e-mail: peta.hruska.1@gmail.com ical modeling of vacancy-hydrogen complexes has been performed for fcc metals, $\mathrm{Pd}$ [13], $\mathrm{Al}$ [14], bcc metals, $\mathrm{Nb}$ [13], Fe [15], as well as hcp metals, e.g. Ti [16]. There is however a lack of experimental data. Positron lifetime (LT) spectroscopy [17] enables characterization of vacancy-hydrogen complexes on the atomic scale. Hydrogen atoms bound to vacancy reduce free volume in the vacancy and cause shortening of positron lifetime. The shortening of positron lifetime for various vacancyhydrogen complexes can be predicted by density functional theory (DFT) calculations and directly compared with experiment. Theoretical modeling of vacancyhydrogen complexes in hcp $\alpha$-Ti was performed in the present work. Lifetimes of positrons trapped at vacancyhydrogen complexes were calculated. Theoretical calculations were compared with experimental study of $\mathrm{H}$ induced defects in Ti by LT spectroscopy.

\section{Theoretical modeling}

Theoretical modeling of vacancy-hydrogen complexes in $\alpha$-Ti was performed using the Vienna ab initio simulation package (VASP) [18] and projected augmented waves pseudo-potentials [19]. The electron exchange and correlation functional was treated with the Perdew-Wang generalized gradient approximation [20]. Plane-wave energy cut-off was fixed to $400 \mathrm{eV}$ for all calculations. A $4 \times 4 \times 4 k$-point mesh generated using the MonkhorstPack scheme [21] was used to sample the Brillouin zone. All calculations were performed on $216 \mathrm{Ti}$ atoms based orthorhombic supercells with dimensions $6 a, 6 a \sqrt{3} / 2$, $3 c$ using the $\alpha$-Ti lattice parameters $a=2.95 \AA$ and $c=4.68 \AA$. In order to simulate vacancy-hydrogen complex a $\mathrm{Ti}$ ion in the center of the supercell was removed and corresponding number of $\mathrm{H}$ atoms was added to the supercell. All ion positions were relaxed in the DFT calculations. The structural optimization was stopped when the forces converged to less than $0.01 \mathrm{eV} / \AA$. The 
zero-point energy $(Z P E)$ of hydrogen was calculated by summing up the vibrational energies of hydrogen normal modes $Z P E=\frac{1}{2} \sum_{i} h \nu_{i}$, where $h$ and $\nu_{i}$ are the Planck constant and the normal vibration frequencies, respectively.

The vacancy formation energy $E_{f}$ was calculated as

$$
E_{f}=E(\mathrm{v})-\frac{N-1}{N} E(\text { bulk }),
$$

where $E(\mathrm{v})$ is the energy of supercell with vacancy, i.e. containing 215 atoms, and $E$ (bulk) is the energy of a perfect (defect-free) supercell containing $N=216 \mathrm{Ti}$ ions.

One should distinguish the formation energy of vacancy-hydrogen complex and the hydrogen trapping energy to the complex. The formation energy of a vacancy associated with $n$ hydrogen atoms $(\mathrm{v}+n \mathrm{H}$ complex) is the amount of energy needed to form $\mathrm{v}+n \mathrm{H}$ complex

$$
\begin{aligned}
& E_{f, \mathrm{v}+n \mathrm{H}}=E(\mathrm{v}+n \mathrm{H})+Z P E(\mathrm{v}+n \mathrm{H}) \\
& -\frac{N-1}{N} E(\text { bulk })-\frac{n}{2}\left(E_{\mathrm{H}_{2}}+Z P E\left(\mathrm{H}_{2}\right)\right),
\end{aligned}
$$

where $E(\mathrm{v}+n \mathrm{H})$ is the energy of a supercell containing $\mathrm{v}+n \mathrm{H}$ complex, $Z P E(\mathrm{v}+n \mathrm{H})$ is the sum of vibrational energies of normal modes of $\mathrm{H}$ atoms in $\mathrm{v}+n \mathrm{H}$ complex, $E_{\mathrm{H}_{2}}$ is the energy of $\mathrm{H}_{2}$ molecule and $Z P E\left(\mathrm{H}_{2}\right)=$ $0.278 \mathrm{eV}$ is $Z P E$ calculated for $\mathrm{H}_{2}$ molecule.

The $\mathrm{H}$ trapping energy $E_{t, \mathrm{v}+n \mathrm{H}}$ is the energy spent when $\mathrm{H}$ atom absorbed in an interstitial position in $\mathrm{Ti}$ lattice is attached to vacancy containing already $n-1$ $\mathrm{H}$ atoms forming $\mathrm{v}+n \mathrm{H}$ complex, i.e. it is negative of the $\mathrm{H}$ binding energy. The $\mathrm{H}$ trapping energy for $\mathrm{v}+n \mathrm{H}$ complex is calculated as

$$
\begin{aligned}
& E_{t, \mathrm{v}+n \mathrm{H}}=E(\mathrm{v}+n \mathrm{H})+Z P E(\mathrm{v}+n \mathrm{H})+E(\mathrm{bulk}) \\
& -E(\mathrm{v}+(n-1) \mathrm{H})-Z P E(\mathrm{v}+(n-1) \mathrm{H}) \\
& -E\left(\mathrm{H}_{i}\right)-Z P E\left(\mathrm{H}_{i}\right),
\end{aligned}
$$

where $E(\mathrm{v}+(n-1) \mathrm{H})$ and $E\left(\mathrm{H}_{i}\right)$ are the energies of a supercell containing $\mathrm{v}+(n-1) \mathrm{H}$ complex and $\mathrm{H}$ absorbed in interstitial position, respectively. $Z P E(\mathrm{v}+(n-1) \mathrm{H})$ and $Z P E\left(\mathrm{H}_{i}\right)$ are corresponding zero point vibrational energies of hydrogen atoms. Note that negative trapping energy means that it is energetically favorable for $\mathrm{H}$ to be trapped in a $\mathrm{v}+(n-1) \mathrm{H}$ complex.

The $\mathrm{H}$ insertion energy into an interstitial site in $\alpha$-Ti lattice, i.e. the formation energy of $\mathrm{H}$ interstitial $E_{f, \mathrm{H}_{i}}$, can be calculated from the relation

$$
\begin{aligned}
& E_{f, \mathrm{H}_{i}}=E\left(\mathrm{H}_{i}\right)+Z P E\left(\mathrm{H}_{i}\right)-E(\text { bulk }) \\
& \quad-\frac{1}{2}\left(E_{\mathrm{H}_{2}}+Z P E\left(\mathrm{H}_{2}\right)\right) .
\end{aligned}
$$

The calculations of positron parameters were performed within so-called standard scheme [22] assuming that positron density is everywhere vanishingly small and not affecting the bulk electron structure. The ground state positron wave function was calculated by solution of a single particle Schrödinger equation

$$
-\frac{\hbar^{2}}{2 m_{0}} \nabla^{2} \psi_{+}(\boldsymbol{r})+V_{+}(\boldsymbol{r}) \psi_{+}(\boldsymbol{r})=E_{+} \psi(\boldsymbol{r}),
$$

where $E_{+}$is the ground state energy eigenvalue. The effective positron potential was constructed as

$$
V_{+}(\boldsymbol{r})=V_{\text {Coulomb }}(\boldsymbol{r})+V_{\text {corr }}\left(n_{-}(\boldsymbol{r})\right),
$$

where $V_{\text {Coulomb }}(\boldsymbol{r})$ is the Coulomb potential produced by the charge distribution of electrons and nuclei. $V_{\text {corr }}\left(n_{-}(\boldsymbol{r})\right)$ is zero positron density limit of the electron-positron correlation potential [22] and $n_{-}(\boldsymbol{r})$ is electron density without presence of positron.

The positron lifetime $\tau$ is determined from the equation

$$
\tau^{-1}=\pi r_{e}^{2} c \int n_{+}(\boldsymbol{r}) n_{-}(\boldsymbol{r}) \gamma\left(n_{-}(\boldsymbol{r})\right),
$$

where $\gamma$ is the enhancement factor accounting for increase of electron density at the site of positron [22]. The electron-positron correlation effects, i.e. $V_{\text {corr }}\left(n_{-}(\boldsymbol{r})\right)$ and $\gamma\left(n_{-}(\boldsymbol{r})\right)$, were treated within the local density approximation using the parametrization by Boronski and Nieminen [23]. Positron lifetime calculations were performed both for relaxed and non-relaxed geometries of vacancy-hydrogen complexes. Electron density was constructed by atomic superposition [24].

\section{Experimental details}

Bulk $\alpha$-Ti samples $(99.7 \%)$ annealed at $1000{ }^{\circ} \mathrm{C}$ for $2 \mathrm{~h}$ in vacuum were studied. Two different procedures were used for $\mathrm{H}$ loading: (i) electrochemical charging at room temperature with a constant current of $20 \mathrm{~mA}$ applied for $240 \mathrm{~h}$ in an electrolyte consisting of $\mathrm{H}_{3} \mathrm{PO}_{4}$ and glycerine in the volume ratio $1: 2$; (ii) gas loading performed at $150^{\circ} \mathrm{C}$ for $100 \mathrm{~h}$ in a high pressure chamber with $\mathrm{H}_{2}$ pressure of 103 bar.

$\mathrm{A}^{22} \mathrm{Na}$ radioisotope with activity of $1.5 \mathrm{MBq}$ deposited on $2 \mu \mathrm{m}$ thick Mylar foil was used as a positron source. LT studies were carried out on a digital spectrometer [26] with time resolution of 145 ps. At least $10^{7}$ positron annihilation events were collected in each LT spectrum. The source contribution was determined using a well annealed reference Ti sample and consisted of two components with lifetimes $\approx 368 \mathrm{ps}$ and $\approx 1.5$ ns and corresponding intensities $\approx 6 \%$ and $\approx 1 \%$.

\section{Results and discussion}

The $\alpha$-Ti lattice contains octahedral (O) and tetrahedral $(\mathrm{T})$ interstitial sites. The calculated $\mathrm{H}$ insertion energy $E_{f, \mathrm{H}_{i}}$ for $\mathrm{O}$ and $\mathrm{T}$ site is -0.57 and $-0.49 \mathrm{eV}$, respectively. Hence, $\mathrm{H}$ absorption in $\alpha$-Ti is an exothermic process for both kinds of interstitial sites but $\mathrm{O}$ sites are energetically slightly more favorable. The same conclusion has been drawn for $\alpha$-Ti in literature [16, 26]. The experimental value of hydrogen insertion energy for $\alpha$-Ti is $-0.54 \mathrm{eV}$ [27]. This value is obviously in good agreement with our calculations. But contrary to theoretical results experimental data indicate that $\mathrm{H}$ absorbed in $\alpha$ Ti lattice prefers $\mathrm{T}$ sites. The reason of this discrepancy is not clear yet but might be caused by a non-negligible 
anharmonic contribution to $Z P E$ which is not considered within the Einstein model.

The formation energy calculated for Ti vacancy $E_{f}=$ $2.09 \mathrm{eV}$ falls into the range $1.96-2.14 \mathrm{eV}$ of values obtained in previous $a b$ initio calculations for monovacancy in $\alpha$-Ti $[16,28,29]$.
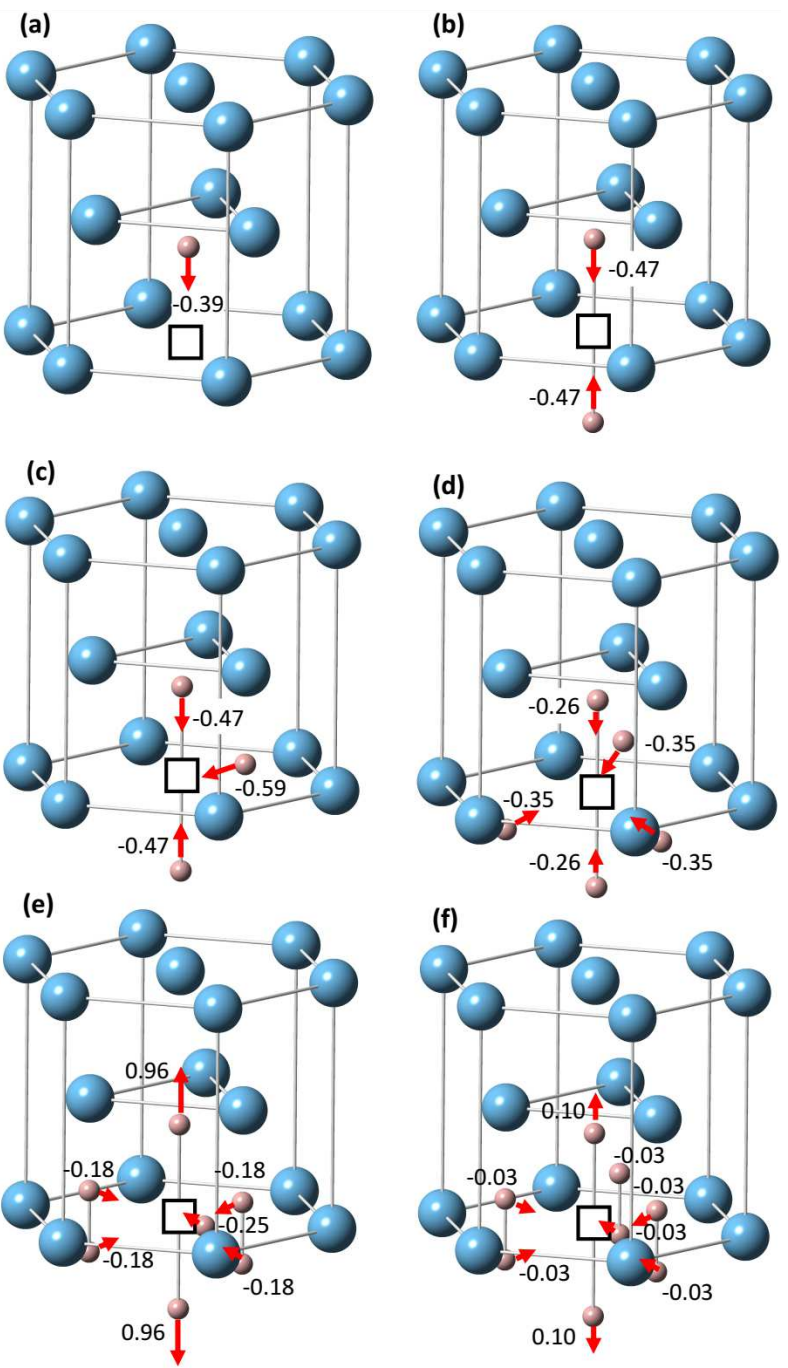

Fig. 1. Schematic depiction of calculated geometries of vacancy-hydrogen complexes: (a) $\mathrm{v}+\mathrm{H},(\mathrm{b}) \mathrm{v}+2 \mathrm{H}$, (c) $\mathrm{v}+3 \mathrm{H}$, (d) $\mathrm{v}+5 \mathrm{H}$, (e) $\mathrm{v}+7 \mathrm{H}$, (f) $\mathrm{v}+8 \mathrm{H}$. Larger and smaller balls show $\mathrm{Ti}$ and $\mathrm{H}$ ions, respectively, open square indicates the center of vacancy. The labels in the figures are calculated displacements of $\mathrm{H}$ atoms from interstitial T-sites in $\AA$.

Figure 1a shows the geometry of $\mathrm{v}+\mathrm{H}$ complex. In the lowest energy configuration $\mathrm{H}$ is displaced from the nearest neighbor (NN) T site towards the vacancy. H trapping in vacancy is energetically favorable since the calculated $\mathrm{H}$ trapping energy is negative, $E_{t, \mathrm{v}+\mathrm{H}}=-0.10 \mathrm{eV}$. The formation energy of $\mathrm{v}+\mathrm{H}$ complex is $E_{f, \mathrm{v}+\mathrm{H}}=1.88 \mathrm{eV}$. Since this value is lower than $E_{f}$ absorbed $H$ reduces the vacancy formation energy in $\alpha$-Ti. The calculated electron ad positron density in the [0001] direction for a plain
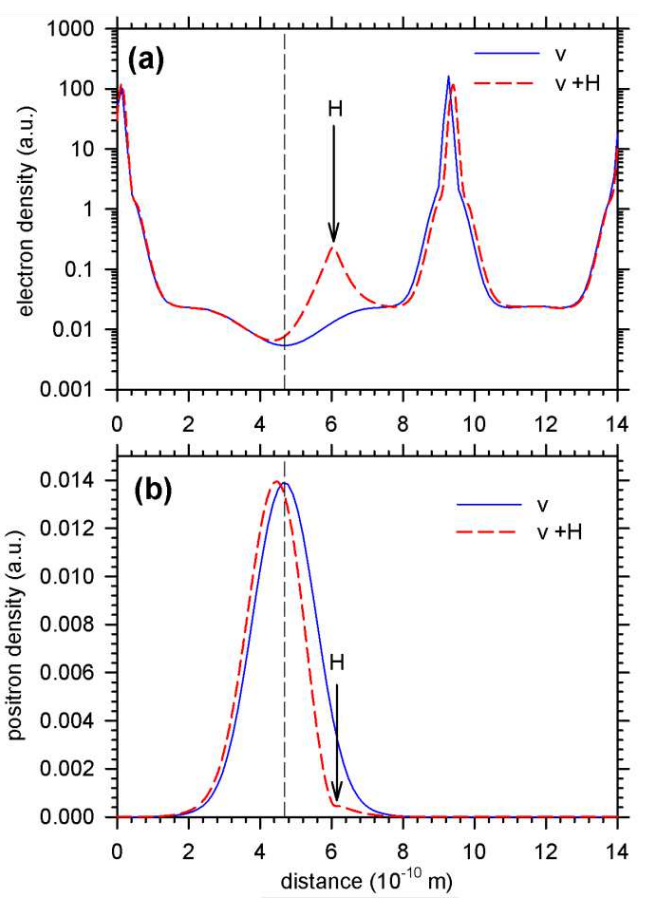

Fig. 2. Calculated (a) electron density and (b) positron density in the [0001] direction for a plain vacancy $\mathrm{v}$ and $\mathrm{v}+\mathrm{H}$ complex. The dashed line shows the centre of vacancy. The position of $\mathrm{H}$ atom is indicated by an arrow.

vacancy and $\mathrm{v}+\mathrm{H}$ complex are plotted in Fig. 2. One can see in the figure that although the positron density is reduced at the site of $\mathrm{H}$ atom there is still a significant overlap of the positron density with the electron density introduced by $\mathrm{H}$ atom associated with the vacancy.

There are 8 interstitial $\mathrm{T}$ sites in the NN positions around the centre of vacancy. In the following calculations $\mathrm{v}+n \mathrm{H}$ complexes were modeled by filling these sites with H. Fully relaxed lowest energy configuration was determined for each complex. As an example Fig. 1b-f shows the lowest energy configurations for $\mathrm{v}+2 \mathrm{H}, \mathrm{v}+3 \mathrm{H}$, $\mathrm{v}+5 \mathrm{H}, \mathrm{v}+7 \mathrm{H}$ and $\mathrm{v}+8 \mathrm{H}$ complexes. The calculated formation energy and $\mathrm{H}$ trapping energy for $\mathrm{v}+n \mathrm{H}$ complexes is plotted in Fig. 3a and b, respectively. The formation energy obviously decreases with increase of number of $\mathrm{H}$ atoms trapped in vacancy up to $5 \mathrm{H}$ atoms. There is almost no difference in formation energies of vacancies associated with 5-7 $\mathrm{H}$ atoms but the formation energy for $\mathrm{v}+8 \mathrm{H}$ complex is significantly higher. The $\mathrm{H}$ trapping energy for $\mathrm{v}+n \mathrm{H}$ complexes remains negative up to $7 \mathrm{H}$ atoms but for $\mathrm{v}+8 \mathrm{H}$ complex it abruptly increases. Hence, vacancies in $\alpha$-Ti are capable of trapping up to $7 \mathrm{H}$ atoms but $\mathrm{v}+8 \mathrm{H}$ complex is energetically unstable and it breaks up due to repulsive $\mathrm{H}-\mathrm{H}$ interaction. The repulsive $\mathrm{H}-\mathrm{H}$ interaction leads also to outward relaxation of $\mathrm{H}$ atoms in $\mathrm{v}+8 \mathrm{H}$ complex, see Fig. 1f.

Note that DFT modeling of vacancy-hydrogen complexes in $\alpha$-Ti were performed by Connétable et al. [16] using similar theoretical approach as in the present work. 

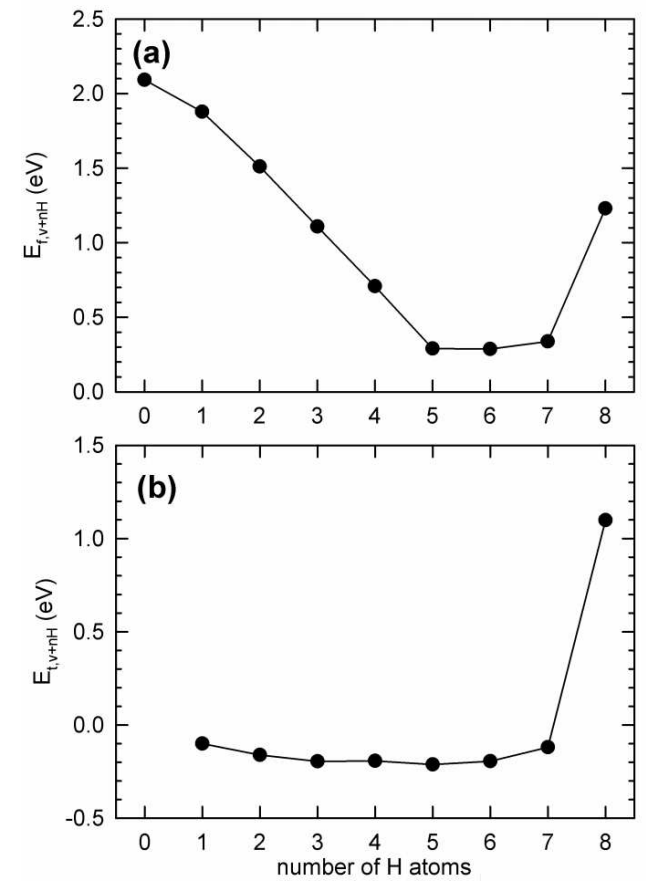

Fig. 3. Calculated (a) formation energy and (b) $\mathrm{H}$ trapping energy for $\mathrm{v}+n \mathrm{H}$ complexes.

The $E_{f}, E_{f, H_{i}}$ values and configurations of $\mathrm{v}-\mathrm{H}$ and $\mathrm{v}-$ $2 \mathrm{H}$ complexes calculated in [16] are similar to those obtained in the present work. But Connétable et al. [16] concluded that the maximum number of $\mathrm{H}$ atoms trapped in vacancy is three. This discrepancy with the present work stems from two factors: (i) the $Z P E$ contribution to the $\mathrm{H}$ trapping energy was not considered in [16] for $\mathrm{v}-n \mathrm{H}$ complexes, c.f. Eq. (22) in [16] and Eq. (3) in the present work. As shown in [30] the zero point vibration energy may constitute a considerable contribution to the $\mathrm{H}$ trapping energy; (ii) Only symmetrical configurations of multiple $\mathrm{H}$ atoms associated with Ti-vacancy were considered by Connétable et al. [16]. But calculations in the present work indicate that non-symetrical configurations of $\mathrm{H}$ atoms located close to NN T sittes around the centre of vacancy represent the lowest energy geometries for $\mathrm{v}+3 \mathrm{H}$ (Fig. 1c), $\mathrm{v}+5 \mathrm{H}$ (Fig. 1d) and $\mathrm{v}+7 \mathrm{H}$ (Fig. 1e) complexes.

Calculated positron lifetimes for relaxed and nonrelaxed $\mathrm{v}+n \mathrm{H}$ complexes are plotted in Fig. 4. From inspection of the figure one can conclude that $\mathrm{H}$ trapped in vacancy reduces lifetime of trapped positrons. For nonrelaxed complexes the decrease of positron lifetime with increase of number of $\mathrm{H}$ atoms is almost linear. Inward relaxation of trapped $\mathrm{H}$ atoms and $\mathrm{NN}$ Ti ions makes the shortening of positron lifetime more pronounced for complexes containing up to $5 \mathrm{H}$ atoms. When the number of trapped $\mathrm{H}$ atoms exceeds 5 some $\mathrm{H}$ atoms and $\mathrm{NN}$ $\mathrm{Ti}$ ions relax outward. As a consequence the lifetime for positrons trapped at $\mathrm{v}+n \mathrm{H}$ complexes containing more than $5 \mathrm{H}$ atoms increases. The outward relaxation becomes most pronounced for $\mathrm{v}+8 \mathrm{H}$ complex which is even

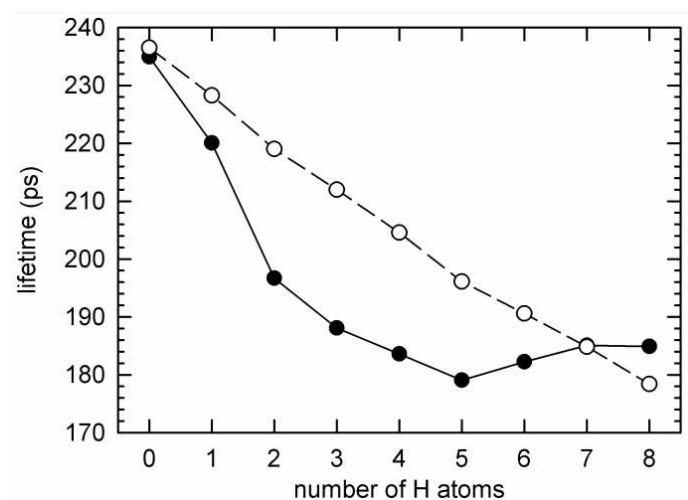

Fig. 4. Calculated lifetimes of positrons trapped at $\mathrm{v}+n \mathrm{H}$ complexes. Relaxed (full points) and non-relaxed (open points) geometries of $\mathrm{v}+n \mathrm{H}$ complexes are compared.

"larger" than the non-relaxed configuration. Hence, one can easy understand that $\mathrm{v}+8 \mathrm{H}$ complex is unstable and breaks up by repulsive $\mathrm{H}-\mathrm{H}$ interaction.

TABLE I

Results of LT measurements of virgin and H-loaded Ti samples.

\begin{tabular}{c|c|c|c|c|c|c}
\hline \hline Sample & $\tau_{1}[\mathrm{ps}]$ & $I_{1}(\%)$ & $\tau_{2}[\mathrm{ps}]$ & $I_{2}(\%)$ & $\tau_{3}[\mathrm{ps}]$ & $I_{3}[\%]$ \\
\hline $\begin{array}{c}\text { virgin } \\
\text { electrochem. }\end{array}$ & $144.6(6)$ & 100 & - & - & - & - \\
$\begin{array}{c}\text { charged } \\
\mathrm{H}_{2} \text { gas } \\
\text { loaded }\end{array}$ & $96(6)$ & $18(1)$ & $170(3)$ & $79(2)$ & $350(20)$ & $3(2)$ \\
& $73(8)$ & $10(1)$ & $171(2)$ & $88(1)$ & $400(60)$ & $2(1)$
\end{tabular}

The results of LT measurements of $\alpha$-Ti samples are collected in Table I. The virgin sample exhibits a single component spectrum with lifetime $\tau_{B}=144.6(6) \mathrm{ps}$, which agrees well with the bulk lifetime for $\alpha$-Ti [31]. Hence, the defect concentration in the virgin sample is very low and virtually all positrons are annihilated in the free state. H-loaded samples exhibit in addition to the free positron component with lifetime $\tau_{1}<\tau_{B}$ two additional components arising from positrons trapped at $\mathrm{H}$ induced defects: a component with lifetime $\tau_{2} \approx 170 \mathrm{ps}$ and dominating intensity and a weak component with longer lifetime $\tau_{3} \approx 350-400 \mathrm{ps}$. Characterization of $\mathrm{H}$ loaded samples by X-ray diffraction [32] revealed that $\mathrm{H}$ loading led to phase transition to $\delta$-hydride $\left(\mathrm{TiH}_{x}\right)$ in the sub-surface region. This is documented in Fig. 5 which shows a micrograph of the cross-section of $\mathrm{H}_{2}$ gas loaded sample. Sub-surface region transformed into $\delta$-hydride is clearly visible. Formation of $\delta$-hydride phase leads to plastic deformation of the sample since volume of the $\delta$ hydride phase is larger than volume of the $\alpha$-Ti matrix. Hence, although the lifetime of the dominating component is relatively close to the calculated lifetime for $\mathrm{v}+5 \mathrm{H}$ complex, positrons in H-loaded samples are annihilated predominantly in dislocations. The longer component with lifetime $\tau_{3}$ can be attributed to vacancy clusters created by agglomeration of $\mathrm{H}$-induced vacancies. $A b$ initio calculations revealed that divacancy consisting of two vacancies located in NN sites is energetically more fa- 


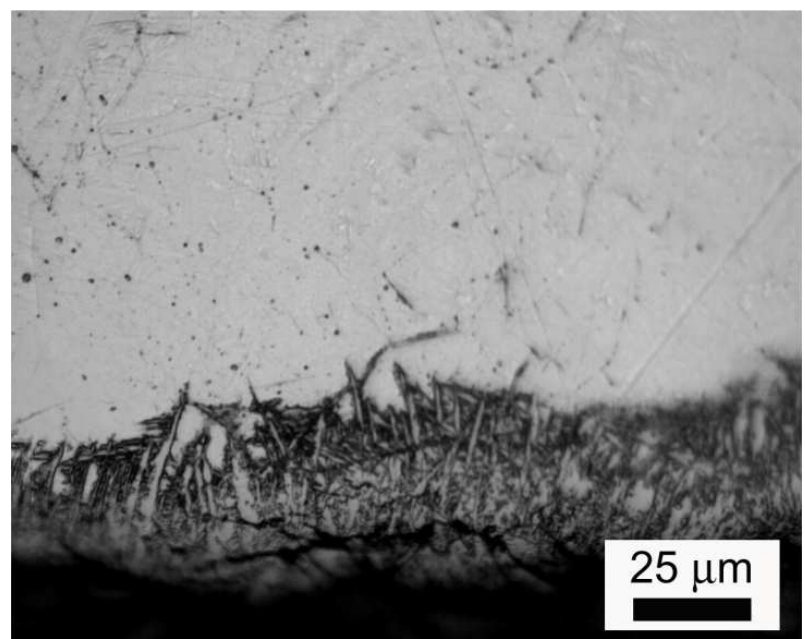

Fig. 5. Optical microscopy image of a cross-section of $\mathrm{H}_{2}$ gas loaded $\alpha$-Ti sample.

vorable than two isolated vacancies. Therefore one can expect that vacancies in Ti tend to agglomerate in order to minimize their energy. Thus, H-loading indeed introduced vacancies but they agglomerated into small clusters which are probably decorated by hydrogen. Hence, the present results showed that in $\alpha$-Ti the phase transition into $\delta$-hydride is energetically more favorable than formation of $\mathrm{H}$ solid solution with enhanced concentration of $\mathrm{v}+n \mathrm{H}$ complexes.

\section{Conclusions}

$A b$ initio theoretical modeling of vacancy-hydrogen complexes in hcp $\alpha$-Ti was performed. Absorbed $\mathrm{H}$ atoms are attracted to vacancies since the energy of $\mathrm{H}$ trapped in vacancy is lower than the energy of interstitial hydrogen. Trapped $\mathrm{H}$ atoms are located close to the nearest neighbor tetrahedral interstitial sites around the centre of vacancy. Up to $7 \mathrm{H}$ atoms can be trapped in vacancy. The lifetime of positrons trapped in vacancyhydrogen complexes decreases with increase of number of $\mathrm{H}$ atoms but when it exceeds 5 it increases again due to outward relaxation caused by repulsive $\mathrm{H}-\mathrm{H}$ interaction. Experimental study of $\mathrm{H}$-loaded Ti samples revealed that $\mathrm{H}$-loading of $\alpha$-Ti introduced dislocations created by phase transition into the $\delta$-hydride and small vacancy clusters formed by agglomeration of $\mathrm{H}$-induced vacancies.

\section{Acknowledgments}

This work was supported by the Czech Science Agency (project P108/12/G043).

\section{References}

[1] Materials Properties Handbook: Titanium Alloys, Eds. R. Boyer, G. Welsch, E.W. Collings, ASM International, 1994.

[2] H. Numakura, M. Koiwa, Acta Metall. 32, 1799 (1984).

[3] E. Tal-Gutelmacher, D. Eliezer, JOM 57, 46 (2005).
[4] A. Pundt, R. Kirchheim, Ann. Rev. Mater. Res. 36, 505 (2006).

[5] R. Kirchheim, Acta Mater. 55, 5129 (2007).

[6] R. Kirchheim, Int. J. Mater. Res. 100, 483 (2009).

[7] G. Lu, E. Kaxiras, Phys. Rev. Lett. 94, 155501 (2005).

[8] Y. Jiraskova, J. Bursik, A. Zemanova, J. Cizek, P. Hruska, O. Zivotsky, Int. J. Hydrogen Energy 42, 6885 (2017).

[9] S. Li, Y. Li, Y.-C. Lo, T.I. Neeraj, R. Srinivasan, X. Ding, J. Sun, L. Qi, P. Gumbsch, J. Li, Int. J. Plastic. 74, 175 (2015).

[10] J. Čížek, I. Procházka, F. Bečvář, R. Kužel, M. Cieslar, G. Brauer, W. Anwand, R. Kirchheim, A. Pundt, Phys. Rev. B 69, 224106 (2004).

[11] Y. Fukai, N. Okuma, Phys. Rev. Lett. 73, 1640 (1994).

[12] C. Freysoldt, B. Grabowski, T. Hickel, J. Neugebauer, G. Kresse, A. Janotti, C.G. van de Walle, Rev. Mod. Phys. 86, 253 (2014).

[13] W. Xing, X.Q. Chen, Q. Xie, G. Lu, D.Z. Li, Y.Y. Li Int. J. Hydrogen Energy 36, 11321 (2014).

[14] M. Benediktsson, K.K.G. Myrdal, P. Maurya, A. Pedersen, J. Phys. Condens. Matter 25, 375401 (2013).

[15] W.A. Counts, C. Wolverton, R. Gibala, Acta Mater 58, 4730 (2010).

[16] D. Connétable, J. Huez, E. Andrieu, C. Mijoule, J. Phys. Condens. Matter 23, 405401 (2011).

[17] R. Krause-Rehberg, H.S. Leipner, in: Positron Annihilation in Semiconductors, Springer-Verlag, Berlin 1999.

[18] G. Kresse, J. Furthmüller, Phys. Rev. B 54, 11169 (1996).

[19] G. Kresse, D. Joubert, Phys. Rev. B 59, 1758 (1999).

[20] Y. Wang, J.P. Perdew, Phys. Rev. B 44, 13298 (1991).

[21] H.J. Monkhorst, J.D. Pack, Phys. Rev. B 13, 5188 (1976).

[22] M.J. Puska, R.M. Nieminen, Rev. Mod. Phys. 66 , 841 (1994).

[23] E. Boroński, R.M. Nieminen, Phys. Rev. B 34, 3820 (1986).

[24] M.J. Puska, R.M. Nieminen, J. Phys. F Met. Phys. 13, 333 (1983).

[25] F. Bečvář, J. Čížek, I. Procházka, J. Janotová, Nucl. Instrum. Methods Phys. Res. A 539, 372 (2005).

[26] Q. Xu, A. Van der Ven, Phys. Rev. B 76, 064207 (2007).

[27] Y. Fukai, in: The Metal-Hydrogen System, SpringerVerlag, Berlin 2005.

[28] O. Le Bacq, F. Willaime, A. Pasturel, Phys. Rev. B 59, 8508 (1999).

[29] D.R. Trinkle, M.D. Jones, R.G. Hennig, S.P. Rudin, R.C. Albers, J.W. Wilkins, Phys. Rev. B 73, 094123 (2006).

[30] Y.-W. You, X.-S. Kong, X.-B. Wu, Y.-C. Xu, Q.F. Fang, J.L. Chen, G.-N. Luo, C.S. Liu, B.C. Pan, Z. Wang, AIP Adv. 3, 012118 (2013).

[31] F. Lukáč, J. Čížek, J. Knapp, I. Procházka, P. Zháňal, R.K. Islamgaliev, J. Phys. Conf. Ser. 674, 012007 (2016).

[32] P. Hruška, J. Čížek, J. Knapp, F. Lukáč, O. Melikhova, S. Mašková, L. Havela, J. Drahokoupil, Int. J. Hydrogen Energy 42, 22557 (2017). 